\title{
Evaluation of matrix-assisted laser desorption ionization-time of flight mass spectrometry in diagnosis of clinical Nocardia species
}

https://doi.org/10.1515/labmed-2018-0180

Received November 16, 2018; accepted April 29, 2019; previously published online May 22, 2019

\section{Abstract}

Background: The routine identification to the species level of Nocardia genus by conventional methods is a fastidious and time-consuming process owing to the limited biochemical reactivity of these microorganisms, often requiring 1 or more days to complete identification. Matrix-assisted laser desorption ionization-time of flight mass spectrometry (MALDI-TOF MS) is a new technology for definitive and rapid species identification.

Methods: We evaluated the MALDI-TOF MS for the identification of 44 clinical isolates of Nocardia species in comparison to $16 \mathrm{~S}$ ribosomal RNA (rRNA) gene sequencing. Nocardia isolates were identified by microbiological examination, phenotypical tests and MALDI-TOF MS and the results were compared by $16 \mathrm{~S}$ rRNA gene sequencing.

Results: Of the 44 Nocardia strains, the identification of 28 isolates was determined with MALDI Biotyper database. According to this, 16 isolates (57.1\%) of the strain log scores were $\geq 2$. Two $(7.1 \%)$ were identified to the species level (log scores of $\geq 2$ ) as Nocardia otitidiscaviarum. The addition of a newly established Nocardia database (16 new Nocardia strains included to the original database) did significantly improve the scores. The results were $43(97.7 \%)$ correct identification to the species level (log scores of $\geq 2$ ).

*Correspondence: Assoc. Prof. Hamit Kaan Müştak, Ankara University, Faculty of Veterinary Medicine, Microbiology Department, Ankara, Turkey, Phone: +903123170315, Fax:+903123186001, E-Mail: kaanmustak@gmail.com. https://orcid.org/0000-0002-3694-1959

Gülşen Hasçelik and Celalettin Uner: Hacettepe University, Faculty of Medicine, Microbiology Department, Ankara, Turkey Markus Kostrzewa: Bruker Daltonics, Bremen, Germany Kadir Serdar Diker: Ankara University, Faculty of Veterinary Medicine, Microbiology Department, Ankara, Turkey
Conclusions: This study showed that the identification of clinical Nocardia isolates by the Bruker MALDI Biotyper is highly reliable, whereas identification rates are generally lower than those for some Gram-negative bacteria and Gram-positive cocci. Based on our data, the identification rates can be improved by validated new database entries and the results can be confirmed with nucleic acid sequence analysis.

Keywords: identification; MALDI-TOF; Nocardia; $16 \mathrm{~S}$ rRNA.

\section{Introduction}

Nocardia species are isolated from clinical specimens, especially those from immunocompromised patients. Infections of Nocardia species range from pulmonary nocardiosis to cutaneous nocardiosis, brain abscess and bacteremia [1]. As there are no comprehensive epidemiological studies related to Nocardia sp. in Turkey, the exact prevalence of the disease is still unknown. However, there are several case reports related to Nocardia spp. associated infections in Turkey [2, 3].

In clinical laboratories, Nocardia species are routinely identified by using Gram and modified acid-fast staining and biochemical tests. These tests are cumbersome, timeconsuming processes and require trained staff [4]. 16S ribosomal RNA (rRNA) gene sequencing is considered to be the gold standard method for definitive identification of Nocardia species [5]. However, these methods are difficult to implement for routine bacterial identification.

Recently, matrix-assisted laser desorption ionizationtime of flight mass spectrometry (MALDI-TOF MS) has been introduced for rapid, accurate, inexpensive and definitive identification of various groups of microorganisms [6-8]. There are several studies on the identification of various groups of microorganisms; however, few studies have investigated the performance of MALDI-TOF MS for Nocardia species [9]. 
The aim of this study was to establish a reference database for clinically relevant Nocardia species and to compare the Bruker MALDI Biotyper system (Bruker Daltonics, Bremen, Germany) to the reference $16 \mathrm{~S}$ rRNA gene sequencing.

\section{Materials and methods}

\section{Patients and bacterial strains}

Forty-four clinical isolates of Nocardia species and one reference strain (Nocardia cyriacigeorgica DSM 44484) were used in this study. The clinical Nocardia isolates had all been isolated from clinical samples of hospitalized adult patients (>18 years of age) between 2002 and 2012 . Among the patients, 25 of them were female and 19 of them were male. Eighteen patients were from Central Anatolia, 12 patients were from the Aegean Region, six were from Marmara, four were from the Black Sea Region and four were from the Southeastern Anatolia Region. Clinical origins of the strains were as follows: 16 strains were isolated from the respiratory tract and two were isolated from the pleural fluid of 18 patients who suffered from pulmonary infection; seven strains were isolated from pus, five strains were from the tissue biopsy samples taken from 12 patients with cutaneous infections; eight strains were from brain abscess, one was from the cerebrospinal fluid samples obtained from nine patients with central nervous infection; among other samples, three of them were taken from patients with conjunctivitis and two of them were obtained from patients who had bacteremia. The study was conducted according to the ethical guidelines.

All of the Nocardia species were stored at $-80^{\circ} \mathrm{C}$ until the initiation of the study. The primary identification of isolates was made by Gram stain, modified acid-fast stain and phenotypical tests (lysozyme resistance, production of arylsulfatase, caseine, xanthine, hypoxanthine, tyrosine and starch hydrolysis, and acid production from rhamnose) and growth at $45^{\circ} \mathrm{C}[10]$.

All strains were subcultured twice on Trypticase soy agar with 5\% sheep blood (BBL, Becton Dickinson Microbiology systems, Cockeysville, MD, USA) and incubated for $48 \mathrm{~h}$ to 5 days at $37{ }^{\circ} \mathrm{C}$ prior to testing. The identification of strains was confirmed by microbiological examination and by biochemical tests.

\section{MALDI-TOF MS}

For the analysis of 44 Nocardia species by the Bruker MALDI Biotyper system, all isolates were cultured on
Trypticase soy agar with 5\% sheep blood (Becton Dickinson Microbiology Systems, Sparks, MD, USA) and incubated for $48 \mathrm{~h}$ at $37^{\circ} \mathrm{C}$. These isolates were extracted using the ethanol-formic acid extraction method [11]. Each sample was spotted in duplicate.

MALDI-TOF MS measurements were performed on a Microflex LT mass spectrometer (Bruker Daltonics GmbH, Bremen, Germany) equipped with a $60-\mathrm{Hz}$ nitrogen laser. MALDI Biotyper 3.1 software program and a reference library of 4612 entries were used to analyze the spectra. For extension of the Bruker MALDI Biotyper database, reference spectra (metaspectra [MSP]) were established based on the Bruker standard procedure. MSP was calculated based on at least 20 mass spectra that passed the quality control successfully. Cut-off values reported by Blosser et al. [9] for the determination of Nocardia isolates were applied to determine the genus-level (1.7000-1.999) or the species-level ( $\geq 2000)$ identification.

\section{DNA extraction and sequence analysis of $16 \mathrm{~S}$ rRNA}

DNA was extracted using the genomic DNA isolation kit (QIAamp DNA Mini Kit; Qiagen) according to the kit protocol. Universal primers: 27F; AGA GTT TGA TCM TGG CTC AG and 1492R; GGT TAC CTT GTT ACG ACT T were used to amplify a 1465-bp product of $16 \mathrm{~S}$ rRNA [12]. The amplified polymerase chain reaction (PCR) products were purified using the QIAquick PCR purification kit (Qiagen) and the BigDye Direct Cycle Sequencing Kit (Applied Biosystems) was used in sequence analyses according to the manufacturer's instructions. After cycle sequencing, amplicons were purified with Sephadex G-50 (Sigma-Aldrich) and sequenced on Applied Biosystems 3500 Genetic Analyzer (Applied Biosystems, CA, USA). All sequences including the positive control strain were analyzed using Sequencing Analysis version 5.3 (Applied Biosystems, CA, USA) and compared with reference sequences in National Center for Biotechnology Information website with BLASTN. Discrepant identification results between the molecular and Bruker MALDI Biotyper methods were repeated twice.

\section{Results}

\section{Phenotypic characterization}

Forty-four clinical isolates were phenotypically characterized as Nocardia species based on colony morphology, 
Gram stain, modified acid-fast stain and resistance to lysozyme. Sixteen Nocardia species were identified to the species level. Twelve strains were identified as Nocardia farcinica and four strains were identified as Nocardia otitidiscaviarum. The residual 28 strains were only identified to the genus level (Table 1).

\section{$16 \mathrm{~S}$ rRNA sequencing of Nocardia strains}

As a reference method all strains were analyzed by $16 \mathrm{~S}$ rRNA gene sequencing. Sequence data of the amplified PCR products showed high similarity to reference sequences of strains in GenBank (National Center for Biotechnology Information [NCBI]) between 97\% and 99\% at the species level. The sequences of 44 Nocardia strains were deposited in GenBank (NCBI) under accession numbers as mentioned in Table 2. According to the results, $N$. cyriacigeorgica was the most frequently isolated species (56.8\%) (Table 1).

\section{MALDI-TOF MS identification of Nocardia strains}

Of the 44 Nocardia strains, the first 28 were evaluated using the MALDI Biotyper 4612 database and only two of them (isolate numbers 16 and 28; N. otitidiscaviarum) were correctly identified at the species level. After DNA sequencing, these 28 ( $N$. cyriacigeorgica [n=15], N. farcinica $[\mathrm{n}=10], N$. otitidiscaviarum $[\mathrm{n}=3]$ ) isolates were added to the database 4612 (Table 2).

Among 44 isolates, 16 of them were not evaluated by the database 4612. However, these isolates were identified by $16 \mathrm{~S}$ rRNA sequencing analysis and one Nocardia asteroides, one Nocardia neocaledoniensis and one Nocardia abscessus species were also added to the database 4612 .

Table 1: Phenotypic and 16S rRNA sequencing results of 44 Nocardia strains.

\begin{tabular}{lrlrr}
\hline \multicolumn{2}{l}{ Phenotypic identification } & & \multicolumn{2}{l}{ 16S rRNA sequencing } \\
\cline { 1 - 1 } Species & Number (\%) & & Species & Number (\%) \\
\hline Nocardia spp. & $28(63.6)$ & & N. cyriacigeorgica & $25(56.82)$ \\
N. farcinica & $12(27.3)$ & & N. farcinica & $12(27.28)$ \\
N. otitidiscaviarum & $4(9.1)$ & & N. otitidiscaviarum & $4(9.09)$ \\
& & N. asteroids & $1(2.27)$ \\
& & N. neocaledoniensis & $1(2.27)$ \\
Total & N. abscessus & $1(2.27)$ \\
& $44(100)$ & & Total & $44(100)$ \\
\hline
\end{tabular}

After these additions, the results were 43 (97.7\%) identified correctly to the species level (log scores of $\geq 2$ ). Only one $N$. neocaledoniensis isolate (isolate number 33) was misidentified as $N$. asteroides in this MALDI Biotyper extended database (Table 3).

\section{Discussion}

In clinical microbiology laboratories, Nocardia are traditionally identified using manual or automated phenotypic and biochemical methods. These methods are generally reliable for species-level identification but are often cumbersome and time-consuming.

Different approaches have been developed to accelerate the identification of clinically significant Nocardia when compared to traditional biochemical phenotyping. These include DNA probe hybridization, PCR and PCRrestriction fragment length polymorphism (RFLP) molecular analysis (PRA), DNA sequencing, pyrosequencing, multilocus sequence analysis and ribotyping [5, 13]. To fully utilize $16 \mathrm{~S}$ rRNA sequencing in clinical microbiology, better guidelines are needed for interpretation of the identification results, and alternative molecular methods [14] are necessary for bacterial species that cannot be identified confidently by16S rRNA gene sequencing alone.

MALDI-TOF MS is a rapid bacterial identification technique that is increasingly used in microbiology laboratories [6]. Previous studies have compared the performance of MALDI-TOF MS systems and DNA sequence analysis techniques for identification of several types of bacterial pathogens; however, only a few studies have investigated the performance of MALDI-TOF MS for Nocardia species [4, 15, 16]. Verroken et al. [4] evaluated the methodology against 43 blind-coded clinical isolates of Nocardia species by phenotypical and fulllength 16S rRNA gene sequencing. Of the studied Nocardia spectra aligned with the MALDI Biotyper database, 19 isolates (44\%) were correctly identified, of which 10 (23\%) were identified to the species level (log scores of $\geq 2$ ) and nine (21\%) were identified to the genus level (log scores between $<2$ and $\geq 1.7$ ) [9]. The addition of their own established Nocardia database to the original database did significantly improve the scores, leading to 38 (88\%) correct identification. The Bruker MALDI Biotyper system failed to identify $N$. abscessus, Nocardia beijingensis, Nocardia brasiliensis, Nocardia carnea and Nocardia veterana, yielded log scores of $<1.7$ and thus could not be identified. In the current study, only N. neocaledoniensis was misidentified as $N$. asteroides. There were 37 Nocardia strains and 35 species in the former database 
with 4612 entries which was called 4612 database. After this study, 28 Nocardia strains and two new species $(N$. asteroides and N. abscessus) were added to the 4612 database and the database increased to 65 Nocardia strains and 37 species. Presently, the MALDI Biotyper database consists of over 7000 entries and called 7000 database, but N. neocaledoniensis still does not exist in this current database.

Correct identification to the species level for 12 different Nocardia species was reported by Farfour et al. [15] to be $91.3 \%$ (42/46 strains) using the Andromas MALDI-TOF MS system with the direct colony method. They concluded

Table 2: Results and scores of 44 Nocardia strains with the DNA sequencing, MALDI Biotyper and MALDI Biotyper extended database.

\begin{tabular}{|c|c|c|c|c|c|c|}
\hline \multirow[t]{2}{*}{ No } & \multirow[t]{2}{*}{ 16S rRNA } & \multirow[t]{2}{*}{ Accession numbers } & \multicolumn{2}{|c|}{ MALDI Biotyper 4612 database } & \multicolumn{2}{|c|}{ MALDI Biotyper extended database } \\
\hline & & & Result & Score & Result & Score \\
\hline 1 & N. cyriacigeorgica & KP268886 & Nocardia sp. & 2.102 & N. cyriacigeorgica ${ }^{\mathrm{b}}$ & 2.647 \\
\hline 2 & N. farcinica & KP279642 & Nocardia sp. & 1.532 & N. farcinica ${ }^{\mathrm{b}}$ & 2.402 \\
\hline 3 & N. cyriacigeorgica & KP268887 & Nocardia sp. & 1.515 & N. cyriacigeorgica ${ }^{\mathrm{b}}$ & 2.314 \\
\hline 4 & N. farcinica & KP279646 & Nocardia sp. & 1.947 & N. farcinica $^{\mathrm{b}}$ & 2.491 \\
\hline 5 & N. farcinica & KP279644 & Nocardia sp. & 1.296 & N. farcinica ${ }^{b}$ & 2.321 \\
\hline 6 & N. cyriacigeorgica & KP268888 & Nocardia sp. & 2.137 & N. cyriacigeorgica ${ }^{\mathrm{b}}$ & 2.507 \\
\hline 7 & N. farcinica & KP279640 & N. brasiliensis & 1.599 & N. farcinica ${ }^{\mathrm{b}}$ & 2.254 \\
\hline 8 & N. farcinica & KP279643 & N. brasiliensis & 1.343 & N. farcinica $^{b}$ & 2.239 \\
\hline 9 & N. cyriacigeorgica & KP268889 & Nocardia sp. & 2.217 & N. cyriacigeorgica ${ }^{\mathrm{b}}$ & 2.47 \\
\hline 10 & N. farcinica & KP279647 & Nocardia sp. & 1.619 & N. farcinica ${ }^{\mathrm{b}}$ & 2.277 \\
\hline 11 & N. cyriacigeorgica & KP268882 & Nocardia sp. & 2.115 & N. cyriacigeorgica ${ }^{b}$ & 2.593 \\
\hline 12 & N. cyriacigeorgica & KP268881 & Nocardia sp. & 2.174 & N. cyriacigeorgica ${ }^{\mathrm{b}}$ & 2.694 \\
\hline 13 & N. cyriacigeorgica & KP268883 & Nocardia sp. & 2.162 & N. cyriacigeorgica ${ }^{\mathrm{b}}$ & 2.396 \\
\hline 14 & N. cyriacigeorgica & KP268884 & Nocardia sp. & 2.255 & N. cyriacigeorgica ${ }^{\mathrm{b}}$ & 2.615 \\
\hline 15 & N. cyriacigeorgica & KP268890 & Nocardia sp. & 2.15 & N. cyriacigeorgica ${ }^{\mathrm{b}}$ & 2.516 \\
\hline 16 & N. otitidiscaviarum & KP268907 & N. otitidiscaviarum & $2.11^{\mathrm{a}}$ & N. otitidiscaviarumb & 2.46 \\
\hline 17 & N. cyriacigeorgica & KP268891 & Nocardia sp. & 2.063 & N. cyriacigeorgica ${ }^{\mathrm{b}}$ & 2.506 \\
\hline 18 & N. cyriacigeorgica & KP268892 & Nocardia sp. & 2.13 & N. cyriacigeorgica ${ }^{\mathrm{b}}$ & 2.637 \\
\hline 19 & N. cyriacigeorgica & KP268893 & Nocardia sp. & 2.203 & N. cyriacigeorgica ${ }^{\mathrm{b}}$ & 2.627 \\
\hline 20 & N. farcinica & KP279648 & Nocardia sp. & 1.555 & N. farcinica ${ }^{\mathrm{b}}$ & 2.288 \\
\hline 21 & N. otitidiscaviarum & KP268908 & N. otitidiscaviarum & 1.804 & N. otitidiscaviarumb & 2.318 \\
\hline 22 & N. farcinica & KP279645 & Nocardia sp. & 1.378 & N. farcinica $^{\mathrm{b}}$ & 2.359 \\
\hline 23 & N. farcinica & KP279649 & Nocardia sp. & 1.559 & N. farcinica $^{\text {b }}$ & 2.285 \\
\hline 24 & N. cyriacigeorgica & KP268894 & Nocardia sp. & 2.119 & N. cyriacigeorgica ${ }^{\mathrm{b}}$ & 2.605 \\
\hline 25 & N. cyriacigeorgica & KP268895 & Nocardia sp. & 2.135 & N. cyriacigeorgica ${ }^{\mathrm{b}}$ & 2.703 \\
\hline 26 & N. cyriacigeorgica & KP268880 & Nocardia sp. & 2.165 & N. cyriacigeorgica ${ }^{\mathrm{b}}$ & 2.583 \\
\hline 27 & N. farcinica & KP279641 & Nocardia sp. & 1.686 & N. farcinica $^{\mathrm{b}}$ & 2.315 \\
\hline 28 & N. otitidiscaviarum & KP268904 & N. otitidiscaviarum & $2.033^{\mathrm{a}}$ & N. otitidiscaviarum ${ }^{b}$ & 2.274 \\
\hline 29 & N. cyriacigeorgica & KP268896 & ND & & N. cyriacigeorgica & 2.073 \\
\hline 30 & N. otitidiscaviarum & KP268905 & ND & & N. otitidiscaviarum & 2.656 \\
\hline 31 & N. cyriacigeorgica & KP268897 & ND & & N. cyriacigeorgica & 2.334 \\
\hline 32 & N. cyriacigeorgica & KP268898 & ND & & N. cyriacigeorgica & 2.355 \\
\hline 33 & N. neocaledoniensis & KP268903 & ND & & N. asteroides $^{\mathrm{b}}$ & 2.015 \\
\hline 34 & N. cyriacigeorgica & KR078341 & ND & & N. cyriacigeorgica & 2.194 \\
\hline 35 & N. cyriacigeorgica & KP268899 & ND & & N. cyriacigeorgica & 2.591 \\
\hline 36 & N. abscessus & KP268876 & ND & & N. abscessus $^{\mathrm{b}}$ & 2.232 \\
\hline 37 & N. farcinica & KP279650 & ND & & N. farcinica & 2.358 \\
\hline 38 & N. farcinica & KP279651 & ND & & N. farcinica & 2.19 \\
\hline 39 & N. cyriacigeorgica & KP268900 & ND & & N. cyriacigeorgica & 2.738 \\
\hline 40 & N. cyriacigeorgica & KP268901 & ND & & N. cyriacigeorgica & 2.177 \\
\hline 41 & N. cyriacigeorgica & KP268879 & ND & & N. cyriacigeorgica & 2.504 \\
\hline 42 & N. cyriacigeorgica & KP268878 & ND & & N. cyriacigeorgica & 2.038 \\
\hline 43 & N. cyriacigeorgica & KP268902 & ND & & N. cyriacigeorgica & 2.606 \\
\hline 44 & N. asteroides & KP268877 & ND & & N. asteroides $^{\mathrm{b}}$ & 2.33 \\
\hline
\end{tabular}

aTwo N. otitidiscaviarum isolates were identified correctly in 4612 database. ${ }^{b}$ Isolates that were added to the database. ND, not done. 
Table 3: Identification results of 44 clinical Nocardia species with the MALDI Biotyper database and extended MALDI Biotyper database.

\begin{tabular}{|c|c|c|c|c|c|c|c|c|}
\hline \multirow[t]{3}{*}{ Log score } & \multicolumn{4}{|c|}{$\begin{array}{r}\text { Isolate results determined with MALDI Biotyper } 4612 \\
\text { database }\end{array}$} & \multicolumn{4}{|c|}{$\begin{array}{r}\text { Isolate results determined with MALDI Biotyper extended } \\
\text { database }\end{array}$} \\
\hline & \multirow[t]{2}{*}{$\%$} & \multicolumn{2}{|c|}{ No. identified $n=28$} & \multirow{2}{*}{$\begin{array}{r}\text { No. } \\
\text { misidentified }\end{array}$} & \multirow[t]{2}{*}{$\%$} & \multicolumn{2}{|c|}{ No. identified $n=44$} & \multirow{2}{*}{$\begin{array}{r}\text { No. } \\
\text { misidentified }\end{array}$} \\
\hline & & Species level & Genus level & & & Species level & Genus level & \\
\hline$\geq 2$ & 57.1 & $2^{\mathrm{a}}$ & 14 & 0 & 100 & 43 & $1^{c}$ & 0 \\
\hline$\geq 1.7$ and $<2$ & 7.2 & 1 & 1 & 0 & 0 & 0 & 0 & 0 \\
\hline$<1.7$ & 35.7 & $2^{b}$ & 8 & $2^{\mathrm{b}}$ & 0 & 0 & 0 & 0 \\
\hline
\end{tabular}

${ }^{a}$ Only two strains were correctly identified as $N$. otitidiscaviarum. ${ }^{\mathrm{b} T w o}$ strains were misidentified as $N$. brasiliensis. ${ }^{\mathrm{c} O n e}$ strain was identified as $N$. neocaledoniensis after 1465 base sequencing.

that for the rapid identification of pathogenic Gram-positive bacilli, these can be identified without an extraction step by MALDI-TOF MS.

Twenty-five clinical strains of Nocardia were studied in the evaluation of MALDI-TOF MS by Xiao et al. [17], but only eight of 25 (32\%) isolates were identified to the genus level and 17 isolates were not identified (68\%). After complementation with the "in-house" database, 95\% were correctly identified to the species level (score $\geq 2.00$ ). Many researchers used the Biotyper MS database in combination with the in-house database that they constructed, thus the identification rate reached to $90 \%$ [18] and $83.1 \%$ [19] from $42 \%$ and 53\%, respectively, in the species level. Pasciak et al. [20] suggested that clinical laboratories processing various clinical strains can upgrade a commercial database to improve and accelerate the results obtained.

Carrasco et al. [21] examined 100 clinical Nocardia strains from different hospitals between 2006 and 2014 and compared the agreement between 16S rRNA full gene and MALDI-TOF MS identification of Nocardia species by using the current commercial database without in-house supplementation. These two methods provided the same identification for $76 \%$ of the strains, and the authors concluded that the Bruker database should be amplified. The MALDI-TOF MS platforms with the Bruker Biotyper library v.4.0 cannot be considered a reliable technique as a routine method for resolving Nocardia spp. identification. All these results show that the performance of the Biotyper is satisfactory using a customized database and in combination with the in-house database that is constructed, but there is still room for diagnostic improvement.

Another commercial MS system is VITEK MS (bioMérieux, Marcy l'Etoile, France). Girard et al. [22] evaluated a set of 164 Nocardia strains using this system, and their database led to $94 \%$ accurate identification to the species level. They concluded that the optimized VITEK MS database with suitable sample preparation protocol enables a rapid, accurate and robust identification of Nocardia spp.
Our analysis evaluated the performance of the Bruker MALDI Biotyper system for identification of Nocardia species that had been confirmed by 16S rRNA gene sequencing. In our study, among 44 Nocardia strains, at first only 28 isolates were evaluated with MALDI Biotyper database officially available at that time (database 4612). Sixteen isolates $(57.1 \%)$ of the strains yielded log scores of $\geq 2$. Only two (4.5\%) were identified to the species level (log scores of $\geq 2$ ) as $N$. otitidiscaviarum. Among these 28 isolates, all of them were added to the database 4612. The rest of the 44 isolates $(n=16)$ were identified by $16 \mathrm{~S}$ rRNA sequencing and three of the Nocardia strains were also added to the database 4612. As a result, the addition of a total of 28 Nocardia strains has significantly improved the scores and after re-evaluation of all 44 strains by the extended database, the results were $43(97.7 \%)$ correct identifications to the species level ( $\log$ scores of $\geq 2$ ).

Several databases and identification strategies have been developed, including the MALDI Biotyper (Bruker Daltonics, Bremen, Germany), the SARAMIS and VITEK MS (bioMérieux, Marcy l'Etoile, France) and the Andromas (Paris, France) systems. Previous reports using the Biotyper system suggested that this technique requires a preliminary extraction step to identify Gram-positive rods [4, 16, 23] but Farfour et al. [15] demonstrated that rapid identification of Nocardia species can be obtained without an extraction step by Andromas MALDI-TOF MS. Schulthess et al. [23] demonstrated that sample preparation using formic acid, compared to the direct transfer preparation method, improved the identification of Grampositive rods by MALDI-TOF MS. Each MALDI-TOF MS identification system has its own specificities, including the way the data was built, the hardware itself and algorithms used to compare the mass spectrum of a sample with those of the database.

In conclusion, this study showed that the identification of several Nocardia species by the Bruker MALDI Biotyper is highly reliable whereas identification rates 
are generally lower than those for some Gram-negative bacteria and Gram-positive cocci. The major limitation of the current study is that relatively few isolates representing a small number of species were examined. Based on our data, the identification rates can be improved significantly by validated new database entries and the results can be confirmed with nucleic acid sequence analysis.

Author contributions: All the authors have accepted responsibility for the entire content of this submitted manuscript and approved submission.

Research funding: This study is supported by the Hacettepe University Scientific Research Projects Coordination Unit (Grant number: 012-D11-101003).

Employment or leadership: None declared.

Honorarium: None declared.

Competing interests: The funding organization(s) played no role in the study design; in the collection, analysis, and interpretation of data; in the writing of the report; or in the decision to submit the report for publication.

\section{References}

1. Ambrosioni J, Lew D, Garbino J. Nocardiosis: updated clinical review and experience at a tertiary center. Infection 2010;38:89-97.

2. Menteş Ö, Zer Y, Balcı I, Özaslan M. First report of pulmonary Nocardia transvalensis infection from Turkey. Pak J Biol Sci 2006;9:2384-6.

3. Sabuncuoğlu H, Açıkgöz ZC, Çaydere M, Üstün H, Keskil S. Nocardia farcinica brain abscess: a case report and review of the literature. Neurocirugia 2004;15:600-3.

4. Verroken A, Janssens M, Berhin C, Bogaerts P, Huang TD, Wauters $G$, et al. Evaluation of matrix-assisted laser desorption ionizationtime of flight mass spectrometry for identification of Nocardia species. J Clin Microbiol 2010;48:4015-21.

5. Brown-Elliott BA, Brown JM, Conville PS, Wallace Jr RJ. Clinical and laboratory features of the Nocardia spp. based on current molecular taxonomy. Clin Microbiol Rev 2006;19:259-82.

6. Seng P, Abat C, Rolain JM, Colson P, Lagier JC, Gouriet F, et al. Identification of rare pathogenic bacteria in a clinical microbiology laboratory: impact of matrix-assisted laser desorption ionization-time of flight mass spectrometry. J Clin Microbiol 2013;51:2182-94.

7. Patel R. A moldy application of MALDI: MALDI-ToF mass spectrometry for fungal identification. J Fungi (Basel) 2019;5:4.

8. Shannon S, Kronemann D, Patel R, Schuetz AN. Routine use of MALDI-TOF MS for anaerobic bacterial identification in clinical microbiology. Anaerobe 2018;54:191-6.

9. Blosser SJ, Drake SK, Andrasko JL, Henderson CM, Kamboj K, Antonara S, et al. Multicenter matrix-assisted laser desorption ionization-time of flight mass spectrometry study for identification of clinically relevant Nocardia spp. J Clin Microbiol 2016;54:1251-8.
10. Brown JM, McNeil MM. Media and methods used for the identification of aerobic Actinomycetes. In: Isenberg HD, Garcia LS, editors. Clinical microbiology procedures handbook, 2nd ed. Washington, DC: ASM Press, 2007:254-66.

11. Alatoom AA, Cunningham SA, Ihde SM, Mandrekar J, Patel R. Comparison of direct colony method versus extraction method for identification of Gram-positive cocci by use of matrix-assisted laser desorption ionization-time of flight mass spectrometry. J Clin Microbiol 2011;49:2868-73.

12. Lane DJ. 16S/23S rRNA Sequencing. In: Stackebrandt E, Goodfellow M, editors. Nucleic acid techniques in bacterial systematics. New York: John Wiley \& Sons, 1991:115-75.

13. McTaggart LR, Richardson SE, Witkowska M, Zhang SX. Phylogeny and identification of Nocardia species on the basis of multilocus sequence analysis. J Clin Microbiol 2010;48:4525-33.

14. Khamis A, Raoult D, La Scola B. Comparison between rpoB and $16 \mathrm{~S}$ rRNA gene sequencing for molecular identification of 168 clinical isolates of Corynebacterium. J Clin Microbiol 2005;43:1934-6.

15. Farfour E, Leto J, Barritault M, Barberis C, Meyer J, Dauphin B, et al. Evaluation of the Andromas matrix-assisted laser desorption ionization-time of flight mass spectrometry system for identification of aerobically growing Gram-positive bacilli. J Clin Microbiol 2012;50:2702-7.

16. Hsueh PR, Lee TF, Du SH, Teng SH, Liao CH, Sheng WH, et al. Bruker Biotyper matrix-assisted laser desorption ionization-time of flight mass spectrometry system for identification of Nocardia, Rhodococcus, Kocuria, Gordonia, Tsukamurella, and Listeria species. J Clin Microbiol 2014;52:2371-9.

17. Xiao M, Pang L, Chen SC, Fan X, Zhang L, Li HX, et al. Accurate identification of common pathogenic Nocardia species: evaluation of a multilocus sequence analysis platform and matrix-assisted laser desorption ionization-time of flight mass spectrometry. PLoS One 2016;11:e0147487.

18. Buckwalter SP, Olson SL, Connelly BJ, Lucas BC, Rodning AA, Walchak RC, et al. Evaluation of MALDI-TOF mass spectrometry for the identification of Mycobacterium species, Nocardia species and other aerobic actinomycetes. J Clin Microbiol 2016;54:376-84.

19. Khot PD, Bird B, Durrant R, Fisher MA. Identification of Nocardia species by matrix-assisted laser desorption/ionization-time of flight mass spectrometry. J Clin Microbiol 2015;53:3366-9.

20. Pasciak M, Dacko W, Sikora J, Gurlaga D, Pawlik K, Miekisiak $\mathrm{G}$, et al. Creation of an inhouse matrix-assisted laser desorption Ionization-time of flight mass spectrometry Corynebacterium database overcomes difficulties in identification of Nocardia farcinica clinical isolates. J Clin Microbiol 2015;53:2611-21.

21. Carrasco G, Caballero D, Garrido N, Valdezate S, Canton R, Saez-Nieto JA. Shortcomings of the commercial MALDI-TOF MS database and use of MLSA as an arbiter in the identification of Nocardia species. Front Microbial 2016;7:542.

22. Girard V, Mailler S, Polsinelli S, Jacob D, Saccomani MC, Celliere $B$, et al. Routine identification of Nocardia species by MALDI-TOF mass spectrometry. Diagn Microbiol Infect Dis 2017;87:7-10.

23. Schulthess B, Bloemberg GV, Zbinden R, Böttger EC, Hombach $M$. Evaluation of Bruker MALDI Biotyper for identification of Gram-positive rods: development of a diagnostic algorithm for clinical laboratory. J Clin Microbiol 2014;52:1089-97. 